\title{
Phasing of ice-sheet and sea-level responses to past climate change
}

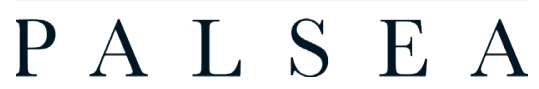

\author{
Alexandra Skrivanek ${ }^{1}$ M.R. Sandstrom ${ }^{2}$ and J. Shakun ${ }^{3}$
}

Playa del Carmen, Mexico, 6-9 November 2017

\begin{abstract}
The exquisitely exposed Last Interglacial (LIG) fossil coral reefs in the Yucatan Peninsula, Mexico, served as the backdrop for the fifth and final meeting of the PALSEA2 (PALeo constraints on SEA level rise 2) working group (Fig.1). The workshop highlighted current research on ice-sheet and sea-level changes, addressed critical gaps in field observations, and assessed the current knowledge regarding causes, rates, and mechanisms of sea-level and ice-sheet dynamics during past warm periods.
\end{abstract}

The five-day program (http://pastglobalchanges.org/ini/wg/palsea2/meetings/127/1715) included 29 presentations, several group discussions, a poster session and field excursions to fossil reefs at Xcaret and limestone caves at Rio Secreto. A major theme was the need to combine existing paleo sea-level and ice-sheet databases into a centralized global compilation with a streamlined user interface. Standardizing, interpreting and assessing the quality of field data were discussed as key components for integration and application by the modeling community. For example, participants considered how sample elevation does not necessarily equate to relative paleo sea level, and the need for clear, systematic descriptions explaining interpretations in paleo databases. Talks explored complications of interpreting sea level from fossil reefs, where accretion is often determined by storm deposition of coral rubble.

Participants also examined the quantification of uncertainty due to glacial isostatic adjustment (GIA) on global sea-level signals in order to reconcile peak LIG sea-level reconstructions. Discussions focused on GIA uncertainty stemming from ice-sheet configurations and 3D Earth-model parameters, as well as the need for additional paleo sea-level data. Suggestions included targeting near-to-intermediate field regions sensitive to GIA, such as the Bahamas, to limit possible ice-sheet configurations, developing adjoint framework methods to efficiently estimate GIA-model parameters, and applying 3D GIA Earth models to investigate model error associated with lateral viscosity variations.

Presentations emphasized the importance of understanding glacial ice-sheet volume and spatial extent prior to the last glacial cycle, which determines GIA effects on LIG sites. New cosmogenic nuclide and sediment provenance techniques have the potential to constrain these glacial ice extents over million-year timescales to orbital timescales, respectively.
Dynamic topography due to mantle convection could have significant effects on the elevation of paleo sea-level indicators, but has substantial vertical uncertainty, leading participants to recommend that larger uncertainty bounds be placed on the current assessment of the peak LIG highstand at 6-9 meters above present global sea level (Dutton et al. 2015). This assessment is consistent with a new, far-field LIG peak sea-level reconstruction from fossil reefs exposed in the Seychelles. Resolving sub-millennial sealevel excursions from LIG deposits remains difficult, considering the vertical uncertainties and complex effects of post-depositional alteration on the interpretation of coral ages. The workshop highlighted new developments regarding the potential for stable oxygen-isotope records from polar ice cores to provide additional constraints on the timing and rate of LIG ice-sheet variability.

Pliocene sea level remains a topic of interest as atmospheric $\mathrm{CO}_{2}$ concentrations were similar to modern values, but constraining global sea level from paleo-shoreline observations and marine geochemical proxies is challenging in light of dynamic topography and diagenesis. Presentations featured a new record of Mid-Pliocene sea-level fluctuations from offshore of New Zealand that offers insight into the pacing and magnitude of ice-sheet variations during this warm period.

Lastly, reconstructions of Holocene ice-sheet stability continue to be refined via multi-proxy

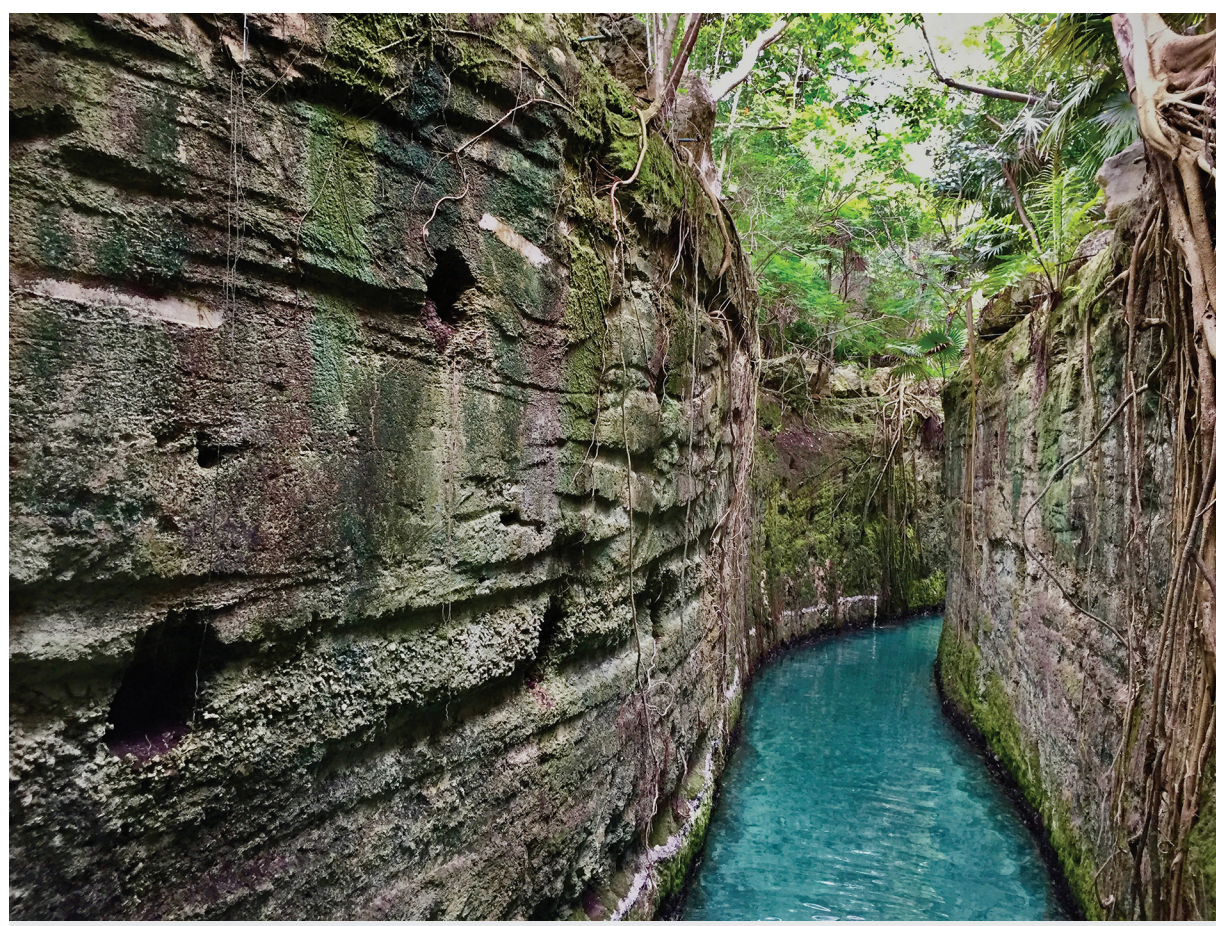

Figure 1: Last Interglacial fossil reefs at Xcaret, Mexico. Image credit: Alexandra Skrivanek. and data-model comparisons as well as the detailed study of coral microatolls. A recent compilation of late-Holocene relative sea-level data and advances in statistical modeling of relative sea-level indicators can support the investigation of factors influencing Holocene sea-level change.

Long-term objectives include improving consistency across the various scientific disciplines in terms of quantifying model accuracy and uncertainty, and endorsing transparency and open-source records for modeling and data acquisitions.

We thank PALSEA2 workshop organizers, namely, Andrea Dutton, Anders Carlson, Glenn Milne, Antony Long and Paul Blanchon, and the supporting organizations: Past Global Changes (PAGES) and the International Union for Quaternary Research (INQUA).

\section{AFFILIATIONS}

Department of Geological Sciences, University of

Florida, Gainesville, USA

2Lamont-Doherty Earth Observatory, Columbia

University, New York City, USA

${ }^{3}$ Department of Earth and Environmental Sciences, Boston College, Chestnut Hill, USA

\section{CONTACT}

Alexandra Skrivanek: lexask@ufl.edu

REFERENCES
Dutton A et al. (2015) Science 349: aaa4019 